\title{
Role of ascorbic acid in the regulation of epigenetic processes induced by Porphyromonas gingivalis in endothelial-committed oral stem cells
}

\author{
Jacopo Pizzicannella ${ }^{1}$. Guya Diletta Marconi ${ }^{2}$. Simone Guarnieri ${ }^{3,4}$ - Luigia Fonticoli $^{5}$. Ylenia Della Rocca ${ }^{5}$.

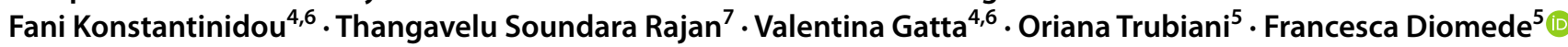

Accepted: 5 July 2021 / Published online: 2 August 2021

(c) The Author(s) 2021

\begin{abstract}
Periodontitis is a common inflammatory disease that affects the teeth-supporting tissue and causes bone and tooth loss. Moreover, in a worldwide population, periodontal disease is often associated with cardiovascular diseases. Emerging studies have reported that one of the major pathogens related to periodontitis is Porphyromonas gingivalis ( $P$. gingivalis), which triggers the inflammatory intracellular cascade. Here, we hypothesized a possible protective effect of ascorbic acid (AA) in the restoration of the physiological molecular pathway after exposure to lipopolysaccharide derived from P. gingivalis (LPS-G). In particular, human gingiva-derived mesenchymal stem cells (hGMSCs) and endothelial-differentiated hGMSCs (e-hGMSCs) exposed to LPS-G showed upregulation of p300 and downregulation of DNA methyltransferase 1 (DNMT1), proteins associated with DNA methylation and histone acetylation. The co-treatment of AA and LPS-G showed a physiological expression of p300 and DNMT1 in hGMSCs and e-hGMSCs. Moreover, the inflammatory process triggered by LPS-G was demonstrated by evaluation of reactive oxygen species (ROS) and their intracellular localization. AA exposure re-established the physiological ROS levels. Despite the limitations of in vitro study, these findings collectively expand our knowledge regarding the molecular pathways involved in periodontal disease, and suggest the involvement of epigenetic modifications in the development of periodontitis.
\end{abstract}

Keywords Periodontitis · Epigenetic modifications · Lipopolysaccharides · Human gingival mesenchymal stem cells $\cdot$ Endothelial differentiation

Francesca Diomede

francesca.diomede@unich.it

1 "Ss. Annunziata" Hospital, ASL 02 Lanciano-Vasto-Chieti, Via dei Vestini, 29, Chieti 66100, Italy

2 Department of Medical, Oral and Biotechnological Sciences, University "G. D’Annunzio" Chieti-Pescara, Via dei Vestini, 31, Chieti 66100, Italy

3 Department of Neuroscience, Imaging and Clinical Sciences, University "G. D’Annunzio" Chieti-Pescara, Via dei Vestini, 31, Chieti 66100, Italy

4 Center for Advanced Studies and Technology (CAST), University "G. D’Annunzio" Chieti-Pescara, Via Luigi Polacchi,19, Chieti 66100, Italy

5 Department of Innovative Technologies in Medicine \& Dentistry, University "G. D’Annunzio" Chieti-Pescara, Via dei Vestini, 31, Chieti 66100, Italy

6 Department of Psychological, Health and Territorial Sciences, University “G. D’Annunzio" Chieti-Pescara, Via dei Vestini, 31, Chieti 66100, Italy

7 Department of Biotechnology, Karpagam Academy of Higher Education, Pollachi Main Road, Eachanari Post, Coimbatore 641021, Tamil Nadu, India

\section{Introduction}

Periodontal disease is widespread, potentially affecting up to $90 \%$ of the world's population. Periodontal diseases, including gingivitis and periodontitis, possess an essential bacterial multifactorial etiology, with the interaction of genetic and environmental factors (Pihlstrom et al. 2005).

Periodontitis is induced by microbial biofilm and caused by host-mediated inflammation, which leads to collateral tissue damage and clinical attachment loss (Hajishengallis et al. 2012). The oral microbiota is composed of more than 700 different species of microorganisms (Aas et al. 2005). Socransky defined the "red complex," which includes Porphyromonas gingivalis, Treponema denticola, and Tannerella forsythia, as the major pathogens responsible for periodontitis progression (Socransky et al. 1998).

In particular, $P$. gingivalis can interfere with host immune responses and alter the symbiosis between the local oral bacteria, 
and can also play a key role in pathogen colonization and periodontitis pathogenesis (Hajishengallis 2014).

Lipopolysaccharide (LPS) is a major component of the cell wall of Gram-negative bacteria, including the periodontal pathogen $P$. gingivalis. LPS is well known for its toxicity and ability to facilitate the host inflammatory response by stimulating Toll-like receptor 4 (TLR4) (Lien et al. 2000), playing a strong pathogenic role in periodontal tissues that leads to a favorable environment for pathogens responsible for sustaining and ultimately causing periodontal disease progression (Papapanou and Susin 2017). Furthermore, common types of periodontal disease and its related infections have been associated with cardiovascular disease (CVD), adverse pregnancy outcomes, stroke, lung disease, and diabetes (Pihlstrom et al. 2005). Uncontrolled periodontal infections can potentially affect vascular endothelial cells with the upregulation of systemic levels of inflammation, and thus contribute to endothelial dysfunction and the onset of atherosclerosis and CVD (Vapaatalo and Mervaala 2001; Aarabi et al. 2018; Priyamvara et al. 2020).

P. gingivalis is gaining recognition as a contributor to CVD such as aortic aneurysm and atherosclerosis (Nakano et al. 2011; Kebschull et al. 2010).

A large number of studies have investigated the mesenchymal stem cell (MSC) response to inflammatory events triggered by different stimuli and treated with antioxidant agents. In recent years, oral tissues such as dental pulp, dental follicle, apical papilla, periodontal ligament, gingiva, and palatine tonsil have been identified as alternative sources for adult MSCs (Andrukhov et al. 2019; Rajan et al. 2017; Bianchi et al. 2017).

Among these, MSCs derived from the human gingiva (hGMSCs) have shown the ability to differentiate into mesengenic lineages and to protect against infectious diseases due to their immunomodulatory properties ( $\mathrm{Li}$ et al. 2014; Hajishengallis 2015; Rajan et al. 2017; Marconi et al. 2020). It must be mentioned here that hMSCs play an active role in the immune response by interacting with natural killer cells, dendritic cells, B lymphocytes and T lymphocytes. In this way, hMSCs prevent improper activation of $\mathrm{T}$ cells and stem the immune response during healing (Papapanou and Susin 2017; Libro et al. 2016a, b; Pisciotta et al. 2020).

Ascorbic acid (AA) is involved in the reduction of reactive oxygen species (ROS) and performs an anti-senescence role (Ballerini et al. 2017). AA, added to cell culture, had been shown to increase cell proliferation and cell pluripotency markers (Frei et al. 1989; Fujisawa et al. 2018), and to promote MSC regeneration of periodontal ligament tissue, reducing inflammation (Wei et al. 2012).

Periodontal disease progression is regulated by bacteria and their products, which may lead to DNA methylation (Diomede et al. 2017; Moore et al. 2013). Epigenetics can be defined as the study of genetic regulatory mechanisms which concern phenotype changes without involving alterations in an individual's DNA sequence. Epigenetic causally related modifications can be responsible for expressing or silencing genes and, as a consequence, determine protein production. These alterations include events such as DNA methylation, histone modification, and microRNA regulation. DNA methylation is a process in which additional methyl groups are added to the DNA, and occurs in regions mostly known as $\mathrm{CpG}$ islands, in which a cytosine nucleotide is located next to a guanine nucleotide, linked by a phosphate. Inserting methyl groups changes the structure of DNA, modifying a gene's interactions with the transcription-associated machinery within a cell's nucleus (Egger et al. 2004). Furthermore, the effects of DNA methylation are often observed in terms of transcriptional modulation; hypermethylation is known to result in a decrease in gene expression, while hypomethylation is usually related to upregulation of transcription (Campos et al. 2016).

Among common oral inflammatory conditions, the presence of epigenetic modifications in proinflammatory cytokines such as Fas-associated death domain protein (FADD), interleukin-12 subunit beta (IL-12B), and interleukin-4 receptor (IL-4R) has been associated with periodontal disease (Schulz et al. 2016). In addition, studies have reported the involvement of DNA methylation events in apical periodontitis-related genes, suggesting epigenetic regulation as a potential regulatory mechanism in the inflammatory response (Mehrazarin et al. 2017). Moreover, considering that AA is an important epigenetic modulator (Lee Chong et al. 2019), we also studied its impact on the promoter methylation status of CCL5 and ICAMI genes as a potential regulatory mechanism in the inflammatory response. Both genes are known to be related to inflammatory response also associated with oral pathological conditions (Wichnieski et al. 2019).

The present study used endothelial-committed oral stem cells as a model to investigate the relationship between periodontal disease and CVD by exposing the cells to lipopolysaccharide derived from $P$. gingivalis (LPS-G).

The aim of the present work was to investigate the antiinflammatory and epigenetic modulatory properties of AA in LPS-G-exposed hGMSCs and e-hGMSCs. Expression of epigenetic markers p300 and DNA methyltransferase 1 (DNMT1), and ROS level were studied.

\section{Materials and methods}

\section{Ethical statement}

The study protocol was approved by the Ethics Committee at the "G. d'Annunzio," Chieti, Italy (n. 266/17). The Department of Medical, Oral and Biotechnological Sciences and 
the Laboratory of Stem Cells and Regenerative Medicine are certified according to the quality standard ISO 9001:2008 (certificate no. 32031/15/S). All patients who were recruited for this study completed and signed an informed consent form. Except following the ethical standards of the institutional and/or national research committee, all procedures act in accordance with the 1964 Declaration of Helsinki and its subsequent reviews.

\section{hGMSC culture establishment}

Patients recruited for this study had to undergo surgical procedures to initiate orthodontic treatment. Gingival tissue was obtained from biopsy during oral surgery procedures. All recruited patients were in good health and did not suffer from oral cavity diseases. After tissue collection, the gingival tissue fragments were crumbled and washed five times with phosphate-buffered saline (PBS) solution (Lonza, Basel, Switzerland). Following these washes, the fragments were placed in a culture dish inside an incubator at $37{ }^{\circ} \mathrm{C}$ with a humidified atmosphere at $5 \% \mathrm{CO}_{2}$ in air. Chemically defined mesenchymal stem cell growth medium (MSCGM$\mathrm{CD}$, Lonza) was used as a culture medium and was changed every 2 days to stimulate the growth of human GMSCs. After 2 weeks of culture, isolated cells were migrated spontaneously from the explants. To evaluate the cell morphology and the capacity to adhere on a plastic substrate, hGMSCs at second passage were seeded on petri dishes and stained with toluidine blue solution, as reported previously (Mazzatenta et al. 2014).

\section{hGMSC characterization by FACS analysis}

After washing in PBS, hGMSCs were analyzed for the expression of CD14, CD34, CD45, CD73, CD90, and CD105. Briefly, cells positive for CD45, CD73, and CD90 were detected with anti-human antibodies conjugated with fluorescein isothiocyanate, while those expressing CD14, CD34 and CD105 were detected with human antibodies conjugated with phycoerythrin. Subsequently, the Fluorescence Activated Cell Sorter (FACS) Star Plus flow cytometry system with CellQuest software (Becton Dickinson, Mountain View, CA, USA) was used for the analysis. All reagents used for flow cytometry were purchased from Becton Dickinson.

\section{Analysis of hGMSC mesenchymal differentiation (colorimetric detection and RT-PCR)}

Since hGMSCs are able to differentiate into mesengenic lines, such as adipogenic and osteogenic commitment, colorimetric detection and reverse transcription polymerase chain reaction (RT-PCR) were used to confirm this differentiation property. Specifically, to induce the adipogenic commitment, the hGMSCs were cultured in a 24-well plate with a density of $2 \times 10^{4}$ cells/well with $500 \mu$ of medium. MSCBM-CD (Lonza) supplemented with $10 \mathrm{mmol} / \mathrm{L}$ dexamethasone, $10 \mathrm{nmol} / \mathrm{L}$ 3-isobutyl-1-methylxanthine, $5 \mathrm{mg} / \mathrm{ml}$ of insulin, and $60 \mathrm{mmol} / \mathrm{L}$ of indomethacin was used to induce adipogenic differentiation for 28 days, with the medium refreshed every 3 days. To evaluate the differentiation, Oil Red O solution (Sigma-Aldrich, Milan, Italy) was used to stain the lipid droplets at the cytoplasmic level. To achieve osteogenic differentiation, the hGMSCs were cultured in a 24-well plate with a density of $2 \times 10^{4}$ cells/well with $500 \mu \mathrm{l}$ of medium. MSCBM-CD (Lonza) supplemented with $10 \mathrm{nmol} / \mathrm{L}$ dexamethasone, $10 \mathrm{nmol} / \mathrm{L}$ beta-glycerophosphate (SigmaAldrich), and $50 \mathrm{mmol} / \mathrm{L}$ AA was used to induce osteogenic differentiation for 21 days. The staining that allowed us to identify the osteogenic differentiation is the Alizarin Red S (Sigma-Aldrich) solution, since it highlights the calcium deposits present in the osteogenic cells (Trubiani et al. 2016). After the staining, the differentiated hGMSCs were visualized under the Leica DM IL inverted light microscope (Leica Microsystems, Milan, Italy). The differentiation was confirmed by RT-PCR for the analysis of the expression of specific osteogenic and adipogenic genes: Runt-related transcription factor-2 (RUNX-2) and alkaline phosphatase (ALP) for osteogenesis commitment, and fatty acid binding protein 4 (FABP4) and peroxisome proliferator-activated receptor $\gamma$ (PPAR $\gamma$ ) for adipogenic differentiation. RT-PCR was performed with the TaqMan Universal PCR Master Mix according to the manufacturer's instructions (Applied Biosystems, Foster City, CA, USA), and $\beta-2$ microglobulin (B2M Hs99999907_m1) (Applied Biosystems) was used for template normalization. To better elucidate the osteogenic differentiation, cells were processed for immunofluorescence staining as reported in the following section "Immunofluorescence analysis and confocal laser scanning microscopy". Primary monoclonal antibody anti-human RUNX2 (1:500; Santa Cruz Biotechnology, Santa Cruz, CA, USA) was used, followed by Alexa Fluor 488 conjugated as secondary antibodies (1:200; Thermo Fisher Scientific, Life Tech., Monza, Italy), and the cells were then observed under a confocal microscope.

\section{hGMSC endothelial differentiation}

To induce endothelial differentiation, the cell culture was stimulated with endothelial growth medium (EGM-2, Lonza) composed of basal endothelial medium-2, R3-insulin-like growth factor-1 (R3-IGF-1), growth supplements containing hydrocortisone, human fibroblast growth factor (hFGF-b), human epithelial growth factor (hEGF), GA-1000, heparin, ascorbic acid, 5\% fetal bovine serum (FBS), and vascular endothelial growth factor 165 (50 ng/ 
ml) (VEGF-165) (EGM-2 Bullet Kit; Lonza). The cells were maintained at $37^{\circ} \mathrm{C}$ with $5 \% \mathrm{CO}_{2}$ by changing the medium every 3 days. After detaching the cells from the monolayer with $0.25 \%$ trypsin-EDTA (Lonza), cells were analyzed after 10 days of stimulation for endothelial differentiation. Differentiation of hGMSCs into e-hGMSCs was observed to begin with a cell confluence of 50-60\%, while hGMSCs, maintained in basal medium, were used as control cells. To evaluate the tube formation capacity, differentiated cells were plated in 12-well culture plates pretreated with $300 \mu \mathrm{l} /$ well of Cultrex ${ }^{\circledR}$ Basement Membrane Extract (Trevigen, Inc., Gaithersburg, MD, USA). The capillary-like tube structures were observed under an inverted light microscope at phase contrast after $4 \mathrm{~h}$ of culture (Pizzicannella et al. 2018). To evaluate the endothelial commitment, e-hGMSCs were processed for immunofluorescence staining as reported in the following section "Immunofluorescence analysis and confocal laser scanning microscopy". Primary monoclonal antibody anti-human VEGF (1:500; Santa Cruz Biotechnology) was used, followed by Alexa Fluor 488 conjugated as secondary antibodies (1:200; Thermo Fisher Scientific), and cells were then observed under a confocal microscope.

\section{Study design}

All the following experiments were performed in triplicate. The present work was carried out with the following groups:

- Untreated hGMSCs, used as negative control (hGMSCs)

- hGMSCs treated for $24 \mathrm{~h}$ with $50 \mu \mathrm{g} \mathrm{mL}^{-1}$ AA (hGM$\mathrm{SCs}+\mathrm{AA})$

- hGMSCs treated for $24 \mathrm{~h}$ with $5 \mu \mathrm{g} \mathrm{mL}^{-1}$ ultrapure LPS-G from $P$. gingivalis (tlrl-ppglps, InvivoGen, San Diego, CA, USA) (hGMSCs + LPS-G)

- hGMSCs co-treated for $24 \mathrm{~h}$ with $50 \mu \mathrm{g} \mathrm{mL}^{-1} \mathrm{AA}$ and $5 \mu \mathrm{g} \mathrm{mL}^{-1}$ LPS-G (hGMSCs + AA/LPS-G)

- Untreated e-hGMSCs, used as negative control (e-hGMSCs)

- e-hGMSCs treated for $24 \mathrm{~h}$ with $50 \mu \mathrm{g} \mathrm{mL}^{-1} \mathrm{AA}$ (e-hGMSCs + AA)

- e-hGMSCs treated for $24 \mathrm{~h}$ with $5 \mu \mathrm{g} \mathrm{mL} \mathrm{m}^{-1}$ ultrapure LPS-G (InvivoGen) (e-hGMSCs + LPS-G)

- e-hGMSCs co-treated for $24 \mathrm{~h}$ with $50 \mu \mathrm{g} \mathrm{mL}^{-1} \mathrm{AA}$ and $5 \mu \mathrm{g} \mathrm{mL}^{-1}$ LPS-G (e-hGMSCs + AA/LPS-G)

\section{Immunofluorescence analysis and confocal laser scanning microscopy (CLSM)}

The sample was fixed with a $4 \%$ solution of paraformaldehyde in 0.1 M PBS (Lonza) (Sinjari et al. 2019). The cells were then permeabilized with $0.5 \%$ Triton X-100 in PBS for 10 min followed by blocking with 5\% skim milk in PBS for $30 \mathrm{~min}$. Subsequently, the cells were incubated for $2 \mathrm{~h}$ at room temperature with the following primary antibodies: anti-p300 (10 $\mathrm{gg} / \mathrm{mL}$; OriGene, Rockville, MD, USA) and anti-DNMT1 (1:100; OriGene). Then, cells were incubated for $1 \mathrm{~h}$ at $37{ }^{\circ} \mathrm{C}$ with Alexa Fluor 568 red fluorescence conjugated as secondary antibodies (1:200; Molecular Probes, Thermo Fisher Scientific). Subsequently, cells were stained for $1 \mathrm{~h}$ with Alexa Fluor 488 phalloidin green fluorescent conjugate (1:400; Molecular Probes) to mark cytoskeleton actin and for $1 \mathrm{~h}$ with TOPRO (1:200; Molecular Probes) to label cell nuclei. Glass coverslips were placed upside down on glass slides and mounted with ProLong Gold Antifade (Molecular Probes). At the end of the staining procedure, samples were observed using a confocal laser scanning microscope (LSM 510 Meta, Zeiss, Jena, Germany), equipped with a Plan-Neofluar oil-immersion objective (Zeiss, $\times 40 / 1.3 \mathrm{NA}$ ). Images were collected using an argon laser beam with excitation lines at $488 \mathrm{~nm}$ and a helium-neon source at 543 and $633 \mathrm{~nm}$. All images were acquired at a resolution of $1024 \times 1024$ pixels at 12 bit (4096 grey values) using ZEN 3.0 SR software (Zeiss).

\section{Western blot analysis}

Proteins from untreated and LPS-G- and/or AA-treated hGMSCs and e-hGMSCs were separated using sodium dodecyl-sulfate polyacrylamide gel electrophoresis (SDSPAGE) followed by western blot analysis (Bio-Rad V3 Western Workflow ${ }^{\mathrm{TM}}$, Milan, Italy).

Membranes were saturated for $120 \mathrm{~min}$ at room temperature in a blocking buffer $(1 \times \mathrm{TBS}, 5 \%$ milk, $0.1 \%$ Tween-20) followed by overnight incubation at $4{ }^{\circ} \mathrm{C}$ with the following primary antibodies: mouse anti-p300 (1:750; OriGene) and mouse anti-DNMT1 (1:750; OriGene). Subsequently, membranes were incubated for $60 \mathrm{~min}$ at room temperature with peroxidase-conjugated anti-mouse secondary antibody (1:5000; Bethyl Laboratories, Montgomery, AL, USA) (Diomede et al. 2016). Enhanced chemiluminescence with the Alliance 2.7 system (Uvitec Ltd, Cambridge, UK) was used to identify and quantify the bands obtained.

\section{ROS labeling}

hGMSCs and e-hGMSCs were seeded in a 35-mm imaging dish ( $\mu$-Dish, ibidi GmbH, Gräfelfing, Germany) and treated for $24 \mathrm{~h}$ in a culture medium containing $5 \mu \mathrm{g} \mathrm{mL}$ LPS-G; (hGMSCs + LPS-G or e-hGMSCs + LPS-G) or $5 \mu \mathrm{g} \mathrm{mL}{ }^{-1}$ LPS-G plus $50 \mu \mathrm{g} / \mathrm{mL}$ ascorbic acid (hGM$\mathrm{SCs}+\mathrm{AA} / \mathrm{LPS}-\mathrm{G}$ or e-hGMSCs + AA/LPS-G) or $50 \mu \mathrm{g} /$ $\mathrm{mL}$ ascorbic acid (hGMSCs + AA or e-hGMSCs + AA); or culture medium alone (hGMSCs, or e-hGMSCs). At the end of expected time, the incubation medium was removed and the cells were washed with normal external solution (NES) containing (in mM) $125 \mathrm{NaCl}, 5 \mathrm{KCl}, 1 \mathrm{MgSO} 4$, 
$1 \mathrm{KH}_{2} \mathrm{PO}_{4}, 5.5$ glucose, $1 \mathrm{CaCl}_{2}, 20$ HEPES, pH 7.4 and incubated with $10 \mu \mathrm{M}$ of $2^{\prime}, 7^{\prime}$-dichlorodihydrofluorescein diacetate (H2DCFDA, Thermo Fisher Scientific, Waltham MA, USA) at $37^{\circ} \mathrm{C}$ in a humidified incubator (for $30 \mathrm{~min}$ ), maintained for all procedures and respective culture media treatments. At the end of dye incubation, the cells were washed with NES and observed in NES alone (hGMSCs or e-hGMSCs), or maintained in NES plus LPS-G, LPS-G and AA, or AA alone. For each condition, confocal microscopy images were randomly acquired using a Zeiss LSM800 microscope (Zeiss), equipped with an Axio Observer D1 inverted microscope and an objective W-Plan-Apo $\times 40 / 1.3$ differential interference contrast (DIC). Excitation was fixed at $488 \mathrm{~nm}$, and emission collection setting of the filter was set over $505-530 \mathrm{~nm}$. The acquisition settings were maintained constant between specimens.

\section{Methylation analysis by pyrosequencing}

DNA was extracted from untreated and treated hGMSCs and e-hGMSCs by LPS and/or AA using the NucleoSpin Tissue kit (Macherey-Nagel, Düren, Germany), following the manufacturer's instructions. DNA quantity and quality were assessed by Qubit 2.0 (Thermo Fisher Scientific).

One hundred nanograms of DNA was bisulfite-converted using the BisulFlash DNA Bisulfite Conversion Easy Kit (EpiGentek Group Inc., NY, USA). PCR amplification of chemokine (C-X-C motif) ligand 5 (CCL5) and intercellular adhesion molecule 1 (ICAMI) promoters was then performed. The PCR mix included the following reagents: KAPA HiFi Uracil Mix (Kapa Biosystems, Merck, Milan, Italy), $0.3 \mu \mathrm{M}$ of each primer, $3 \mu \mathrm{L}$ of converted DNA, and nuclease-free water to a final volume of $50 \mu \mathrm{L}$. Specific sets of primers for PCR amplification and sequencing were designed using specific software (PyroMark Assay Design version 2.0.01.15). Primer sequences (see Table 1) were designed, when possible, to hybridize with $\mathrm{CpG}$-free sites to ensure methylation-independent amplification. Pyrosequencing reactions were run on a PyroMark Q96ID (Qiagen, Hilden, Germany), and $\mathrm{CpG}$ methylation analysis was conducted using PyroMark CpG software (Qiagen).
A triplicate was generated for each PCR using bisulfiteconverted DNA from three different conversion reactions.

The methylation for each amplicon was calculated as the median of methylation status of each analyzed CpG. Differences in methylation pattern across samples and controls were calculated using one-way analysis of variance ANOVA, considering $p$ values $<0.05$ as significant.

\section{Statistical analysis}

GraphPad Prism software (version 5.01, GraphPad Software, San Diego, CA, USA) was used for the statistical analysis. The data were analyzed using one-way ANOVA followed by Tukey's post hoc test. $p<0.05$ was considered statistically significant. Data were expressed as the mean \pm SD. All data were collected for at least three independent experiments.

\section{Results}

\section{hGMSC characterization}

hGMSCs were characterized following the established criteria of the International Society for Cellular Therapy to define their multipotent mesenchymal profile. hGMSCs able to differentiate into osteogenic and adipogenic lineages were maintained under proper culture conditions for 21 and 28 days, respectively. Cells were positive for adipo Oil Red and Alizarin Red staining, to determine the lipid vacuoles at the cytoplasmic level and calcium deposits (Fig. 1b, c). Undifferentiated cells showed a typical fibroblast-like morphology, and they are adherent on a plastic substrate (Fig. 1a).

RT-PCR data showed an increase in the mRNA expression of RUNX-2 and ALP in osteogenic committed cells, and FABP4 and PPAR $\gamma$ in the adipogenic differentiated samples when compared to the basal cells (Fig. 1d, e). Cytofluorimetric determination showed a positivity for $\mathrm{CD} 73,90$, and 105, while cells were negative for typical hematopoietic markers, as CD14, 34, and 45 (Fig. 1f). Osteogenic differentiated cells were positive to RUNX-2 staining, the marker is localized at the nuclear level, as demonstrated by the confocal laser scanning microscopy image (Fig. S1).

Table 1 Pyrosequencing primer sequences

\begin{tabular}{llc}
\hline Genes & Primer sequence & Sequence to analyze \\
\hline CCL5-Hsa-F & 5'-[Btn]AGAGTAGTGAGGGAGAGATAGA-3' & ATTAACCRATATCATAAATAAAATTAC \\
CCL5-Hsa-R & 5'-[AAACATCCCCTAAAAAAAATTTCCA-3' & ACAAAACRAAAAAAAAAACTAAAATAA \\
CCL5-Hsa-Seq & 5'-CCAAAATAACAACCAAAC-3' & CCTCC \\
ICAM1-Hsa-F & 5'-[Btn]-TTTTTTTTTTGGGAGTTGTAAAG-3' & RAACATTTCTCRAAAAACRACCAAAA \\
ICAM1-Hsa-R & 5'-ACTAAAAAATACCCCTCCCCCTAA-3' & \\
ICAM1-Hsa-Seq & 5'-TCCACACCTAACTAACAC-3' \\
\hline
\end{tabular}



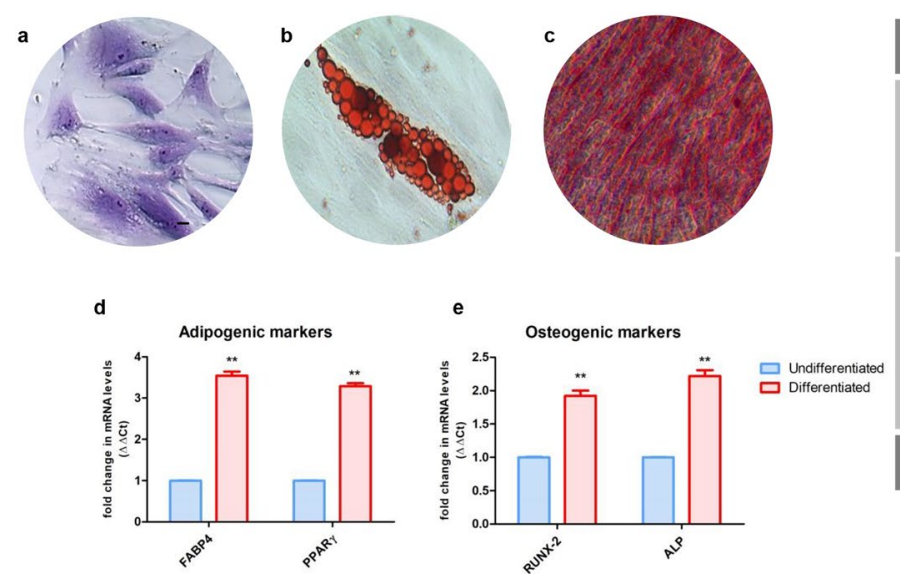

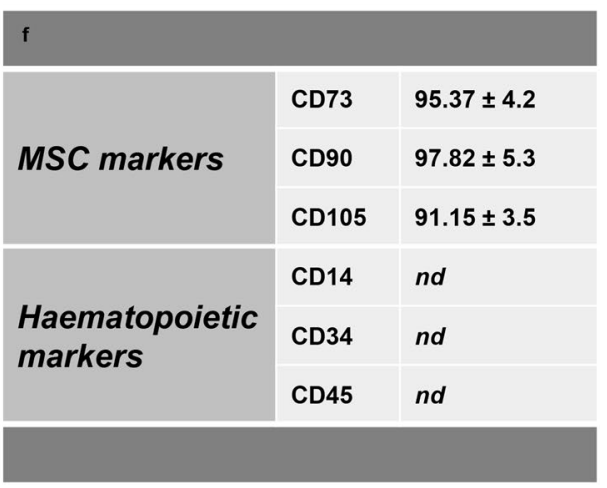

differentiation of hGMSCs: alizarin red staining of calcium deposition. d RT-PCR graph of adipogenic markers. e RT-PCR graph of osteogenic markers. $\mathbf{f}$ The table summarizes the data obtained from the flow cytometric analysis. Scale bar $=20 \mu \mathrm{m}$. $n d$ not detectable. $(N=5)$. Values are expressed as mean $\pm \mathrm{SD}$

by light microscopy following a time course. After $1 \mathrm{~h}$ of culture, cells began to aggregate with each other by the establishment of connections with cytoplasmic processes (Fig. 2a), and after $4 \mathrm{~h}$ of culture, a mesh-like structure became a finer and denser tubular network (Fig. 2b). The

Endothelial-committed hGMSCs were cultured on dishes covered with Cultrex gel solution. These cells were observed
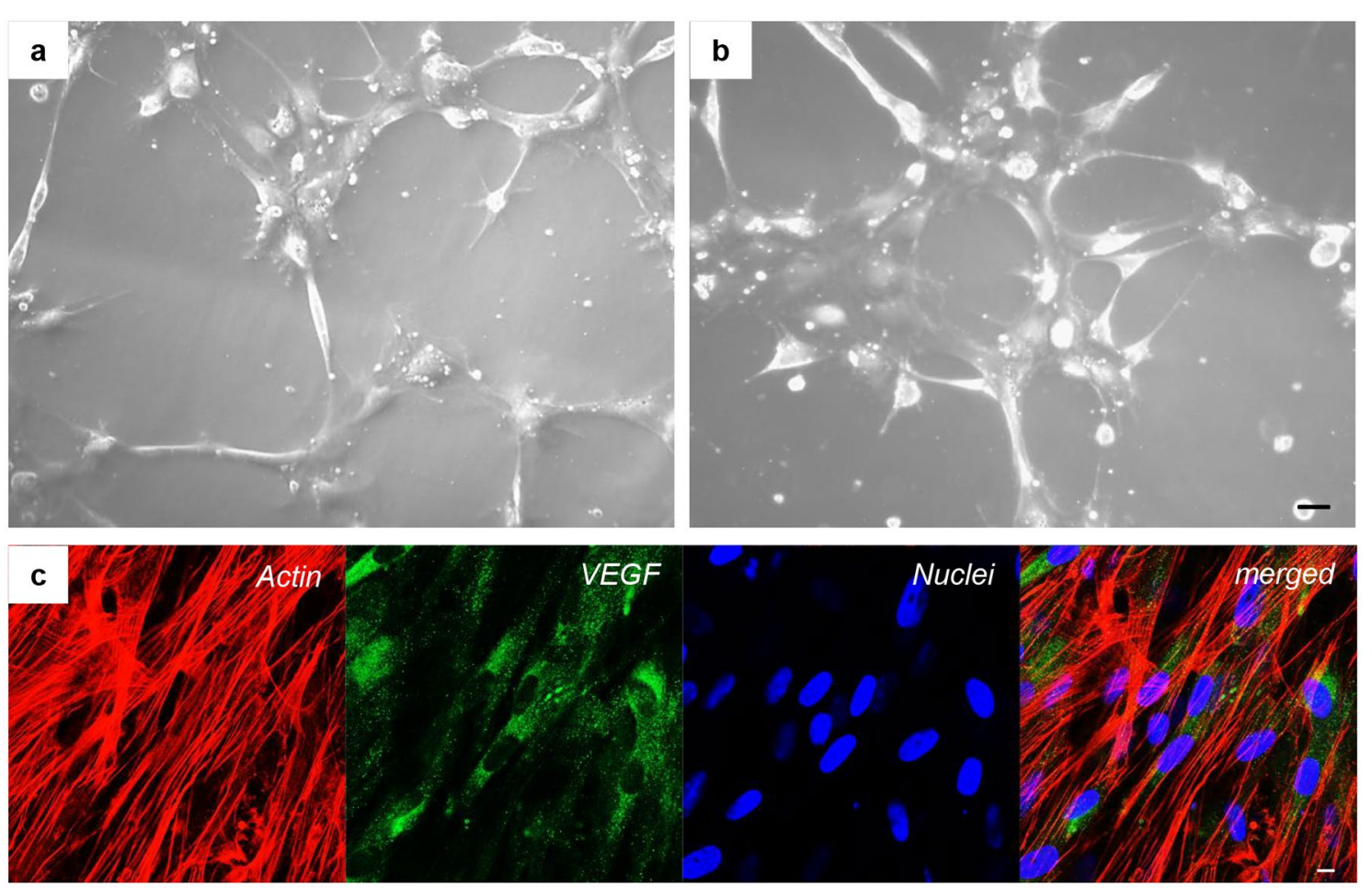

Fig. 2 Time-lapse images of e-hGMSCs during tube formation. a The e-hGMSCs seeded on the Cultrex had begun to aggregate each other $(1 \mathrm{~h})$. b The cells were connected with cytoplasmic processes, devel- oping a dense network (4 h). Bar $=20 \mu \mathrm{m}$. c Immunofluorescence staining of e-hGMSCs showed the positivity for VEGF antigen. Red: cytoskeleton actin. Blue: nuclei. Green: VEGF. Bar $=10 \mu \mathrm{m}$ 
evaluation of the impact of the differentiation medium kit exposure on hGMSCs to induce the endothelial transition (e-hGMSCs) was performed by examining the typical endothelial markers using VEGF immunofluorescence staining (Fig. 2c).

\section{LPS-G-induced modulations in p300 and DNMT1}

We examined whether LPS-G influenced the levels of p300 and DNMT1 in hGMSCs and e-hGMSCs. Immunofluorescence images by confocal microscopy system showed increased expression of nuclear p300 in response to LPS-G stimuli. On the other hand, DNMT1 expression was decreased in the hGMSCs and e-hGMSCs treated with LPS-G when compared to the untreated cells. The treatment with AA alone showed no differences in the expression of p300 and DNMT1 in hGMSCs and e-hGMSCs when compared to the untreated samples. Co-treatment with AA + LPS-G showed an effect in the reduction of p300 expression and an increased expression of DNMT1 when compared to the LPS-G-treated cells (Figs. 3, 4, 5, 6 ). These results were further confirmed by western blot analysis (Fig. 7).

\section{ROS localization}

ROS induced by LPS-G was visualized in hGMSCs and e-hGMSCs loaded with the cell-permeant ROS probe $2^{\prime}, 7^{\prime}$-dichlorodihydrofluorescein diacetate $\left(\mathrm{H}_{2}\right.$ DCFDA) (Fig. 8). After incubation, the probe was permeated into the cell and spread homogeneously inside the cytoplasm and organelles, where the hydrolysis of the acetate groups of $\mathrm{H}_{2}$ DCFDA was catalyzed by intracellular esterase. This resulted in the conversion of non-fluorescent $\mathrm{H}_{2}$ DCFDA into the fluorescence-emitting $\mathrm{H}_{2} \mathrm{DCF}$, which was transformed in the highly fluorescent molecule $2^{\prime}, 7^{\prime}$-dichlorofluorescein (DCF) by ROS. Images were acquired in live cells by means of confocal microscopy, as shown in Fig. 8. In the control conditions (hGMSCs and e-hGMSCs), cells showed a low and diffused fluorescence signal, while an increase

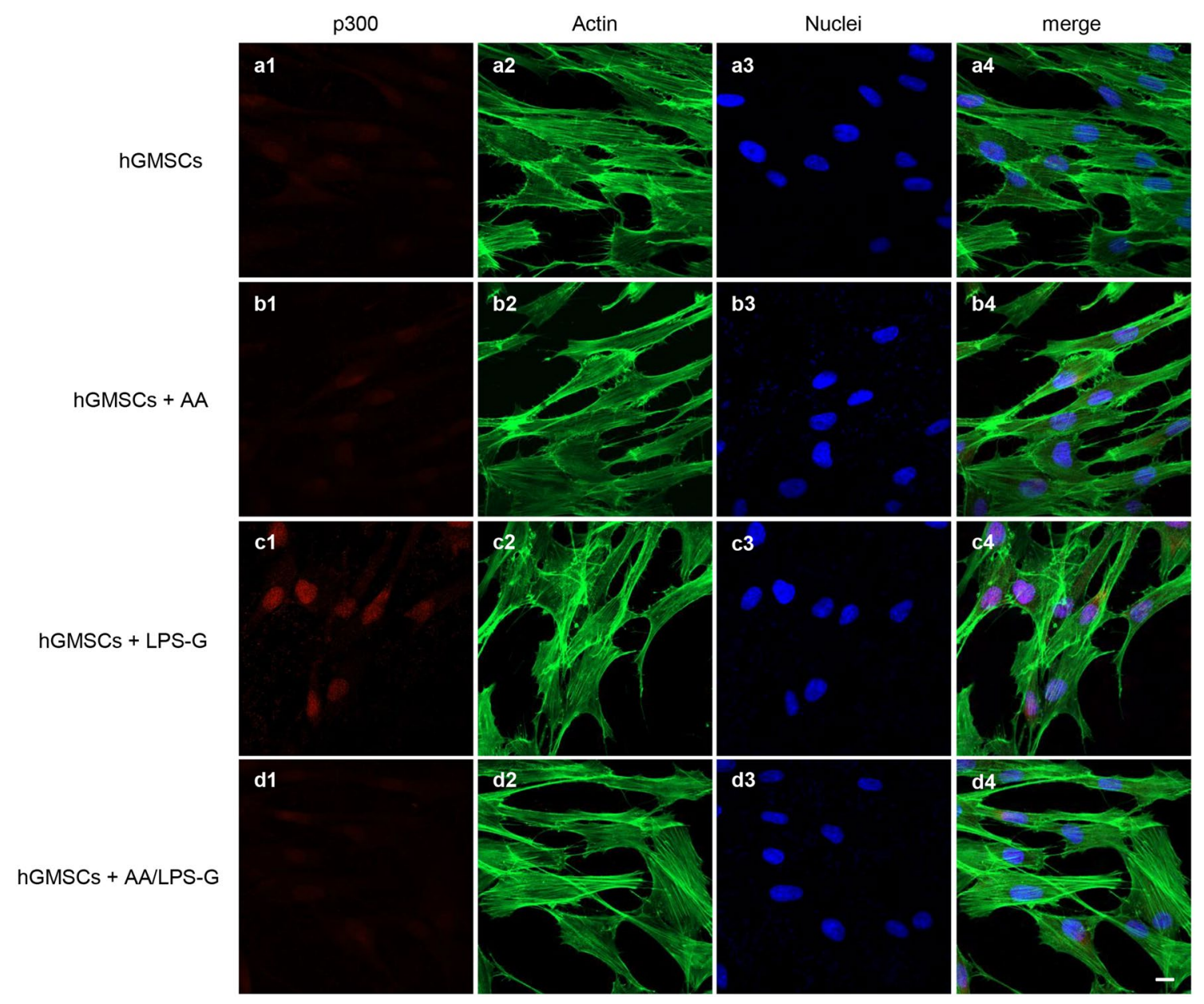

Fig. 3 Immunofluorescence analysis of p300 expression in hGMSCs. a1-a4 Untreated hGMSCs. b1-b4 hGMSCs treated with AA. c1-c4 hGMSCs treated with LPS-G. d1-d4 hGMSCs co-treated with LPS-G and AA. Scale bar $=10 \mu \mathrm{m}$ 


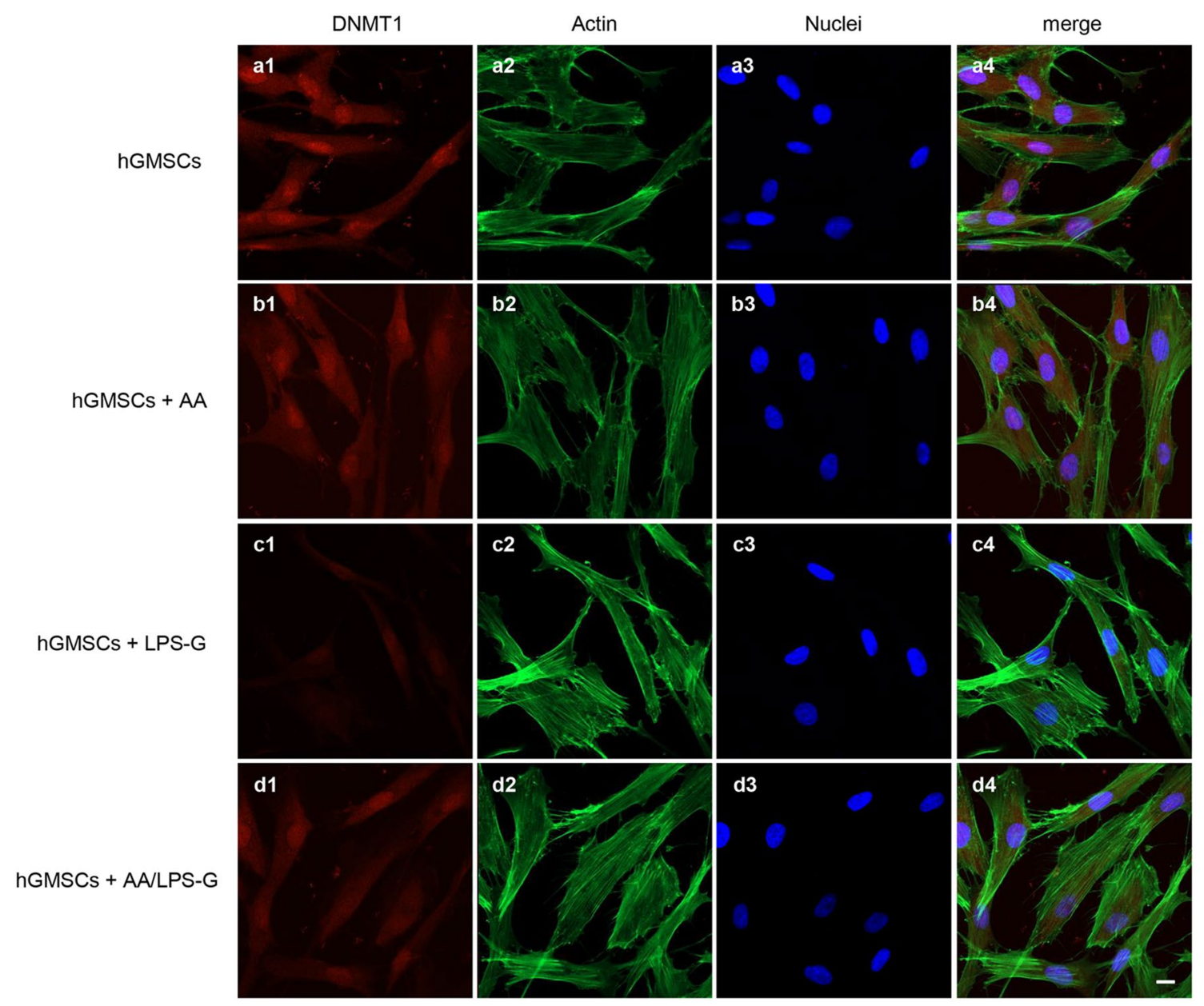

Fig. 4 Immunofluorescence analysis of DNMT1 expression in hGMSCs. a1-a4 Untreated hGMSCs. b1-b4 hGMSCs treated with AA. c1-c4 hGMSCs treated with LPS-G. d1-d4 hGMSCs co-treated with LPS-G and AA. Scale bar $=10 \mu \mathrm{m}$

in ROS production was clearly evident in LPS-G-treated hGMSCs and e-hGMSCs. Interestingly, while in the hGMSCs + LPS-G the emitted fluorescence signal maintained a diffused pattern of emission, in the e-hGMSCs + LPS-G the increased fluorescence appeared to be more strongly localized in a "spaghetti-like" area which possibly denoted the mitochondrial structures (see arrow in Fig. 8, e-hGMSCs + LPS-G). However, addition of AA considerably reduced the LPS-G-induced ROS production in hGMSCs and e-hGMSCs.

\section{Methylation levels of CpGs in CCL5 and ICAM1}

Following statistical analysis of PyroMark Q96ID-generated data using the ANOVA test, $p$-values less than 0.05 were considered significant. No differences in methylation patterns across untreated and treated hGMSCs and e-hGMSCs by LPS-G and/or AA were observed in CCL5 and ICAMI gene promoters (data not shown).

\section{Discussion}

Oral microbiota and their products cause changes in epigenetic mechanisms and trigger inflammation, which results in the development and progression of periodontitis. When left untreated, the oral pathogens increase the proinflammatory mediators distant from their site, which leads to the development of periodontitis-associated arterial disease and CVD.

In the present study, we cultured the hGMSCs from gingival tissues of healthy patients. These cells showed expression of stem cell markers CD73, CD90, and CD105, while the cells were negative for hematopoietic markers CD14 and CD34, as previously demonstrated (Gugliandolo et al. 2018), other than the capacity to differentiate into osteogenic and adipogenic commitment as demonstrated by histochemistry staining and gene expression data. To relate the effect of LPS-G on endothelial cells, endothelial-differentiated cells derived from hGMSCs (e-hGMSCs) were studied. As previously reported, the angiogenic potential of stem cells derived from dental pulp, gingival fibroblasts, and tooth germ are 


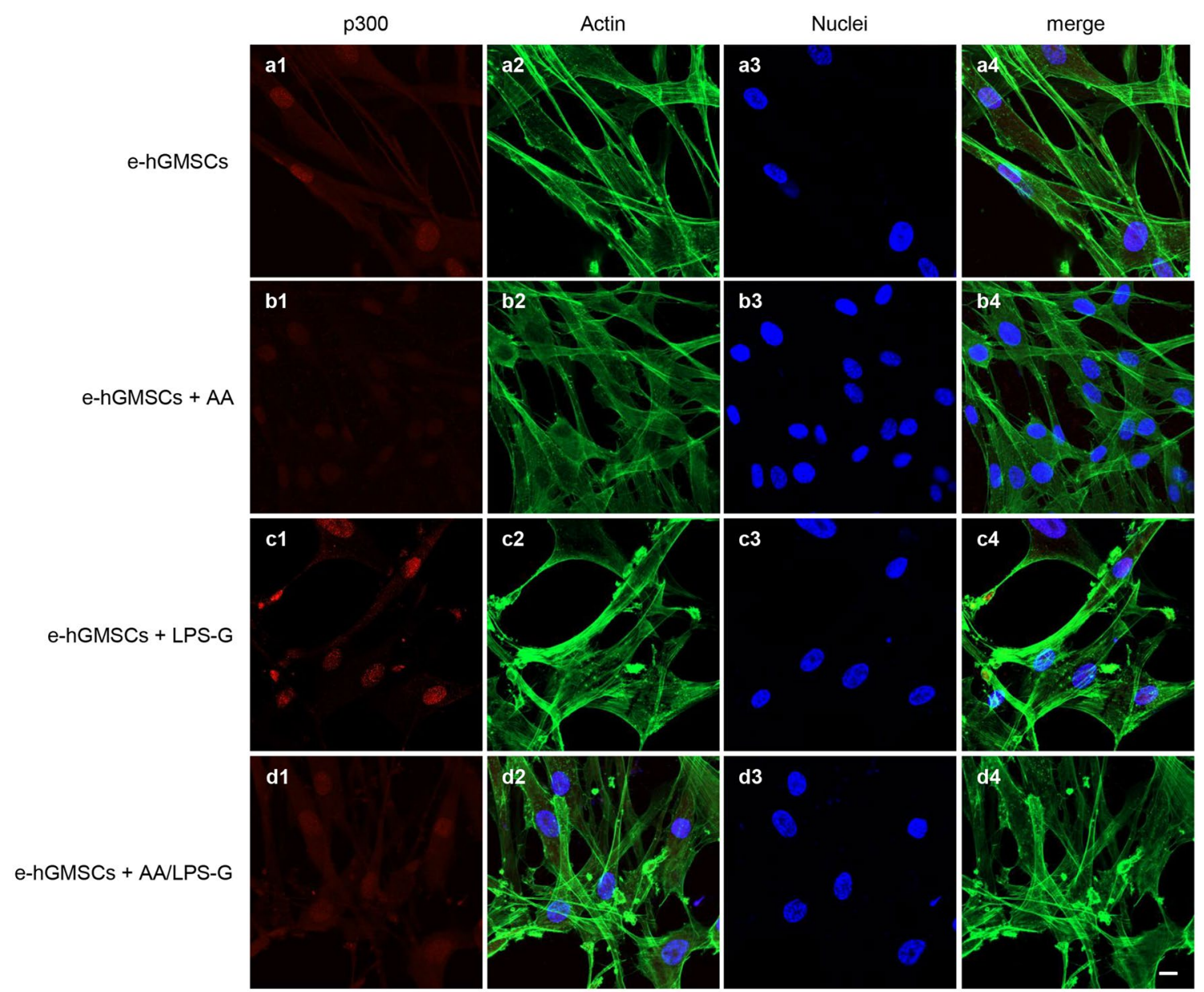

Fig. 5 Immunofluorescence analysis of p300 expression in e-hGMSCs. a1-a4 Untreated e-hGMSCs. b1-b4 e-hGMSCs treated with AA. c1-c4 e-hGMSCs treated with LPS-G. d1-d4 e-hGMSCs co-treated with LPS-G and AA. Scale bar = $10 \mu \mathrm{m}$

able to generate endothelial tissues (Diomede et al. 2020; Pizzicannella et al. 2019). Accordingly, hGMSCs were induced to endothelial commitment, and their differentiation was evaluated by means of positivity to VEGF expression as demonstrated by immunofluorescence images and to CD31 expression, as reported previously (Pizzicannella et al. 2018). Moreover, endothelial differentiation is defined by the capacity to form a tubular structure (Barachini et al. 2014). In our study, the induced e-hGMSCs showed a network structure after seeding on Cultrex, demonstrating a capillary-like complex.

After successful establishment of hGMSCs and e-hGM$\mathrm{SCs}$, the cells were exposed to LPS-G to trigger the changes in epigenetic mechanisms. Expression of histone acetyltransferase p300 and DNMT1 were investigated by immunofluorescence staining. We found that the level of p300 was increased in hGMSCs and e-hGMSCs after LPS-G treatment. Earlier studies have reported that $P$. gingivalis and Fusobacterium nucleatum are able to activate p300/ cyclic AMP response element-binding protein (CBP) in oral epithelial cells (Liu et al. 2008). Although the precise role of p300 in periodontal disease maintenance and progression remains unclear, it is reported to regulate inflammatory NFkB signaling (Vanden Berghe et al. 1999). On the other hand, the expression of DNMT1 was decreased in LPS-Gtreated hGMSCs and e-hGMSCs. Similar data were reported in a previous study where LPS exposure reduced the expression of DNMT1 in oral epithelial cells (Martins et al. 2016). LPS-G-mediated changes in the level of p300 and DNMT1 may activate the production of inflammatory mediators, which may contribute to the progression and maintenance of inflammation (Pizzicannella et al. 2021). Then, we studied the protective anti-inflammatory role of AA in LPS-Gtreated hGMSCs and e-hGMSCs. AA is a powerful reducing agent and antioxidant. The efficacy of AA in reversing periodontitis and chronic gingival inflammation has been demonstrated (Chapple and Matthews 2007; Yussif et al. 2016; Diomede et al. 2019). In our study, we observed that the proinflammatory epigenetic changes caused by LPS-G were efficiently nullified by AA. Immunofluorescence 


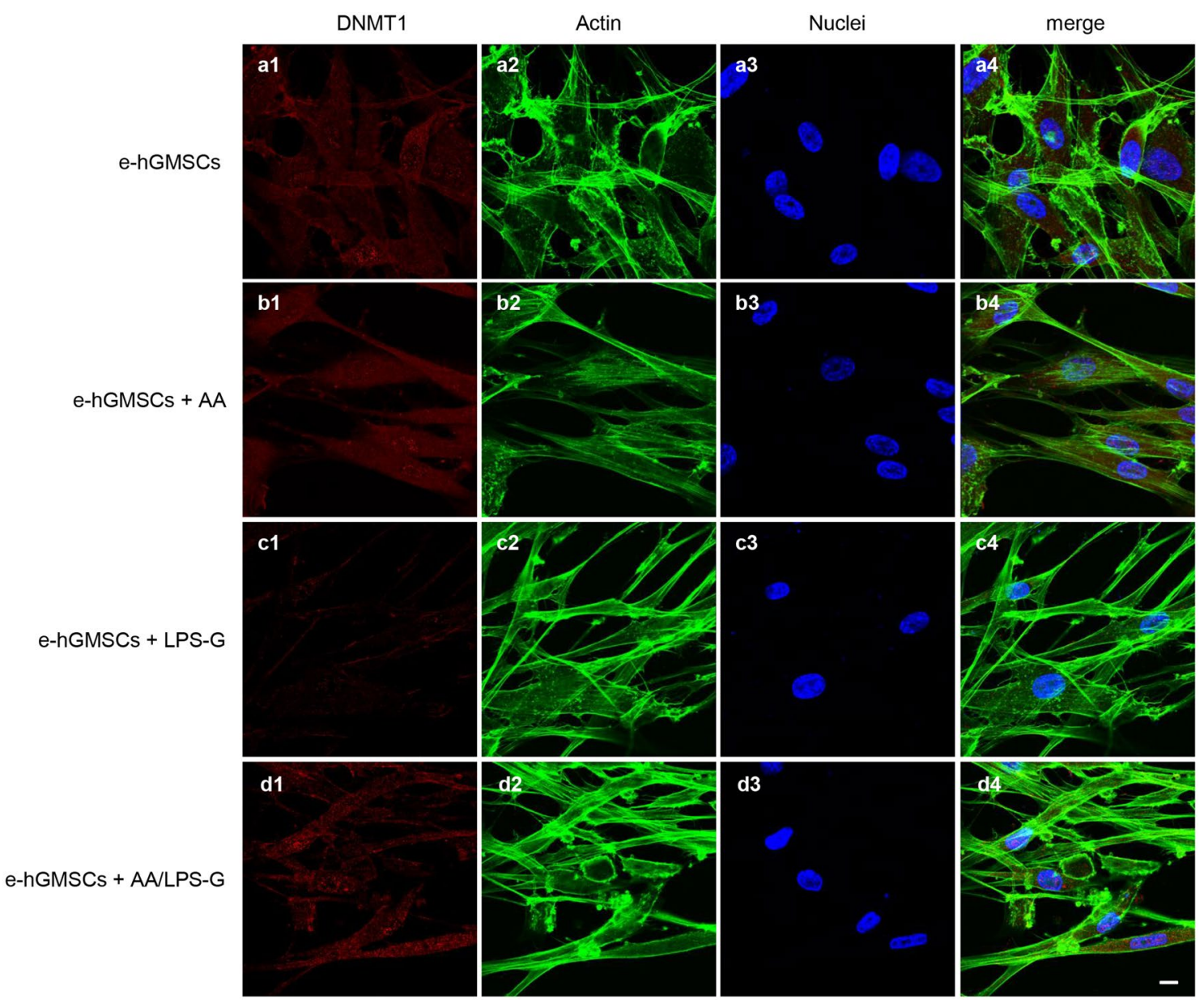

Fig. 6 Immunofluorescence analysis of DNMT1 expression in e-hGMSCs. a1-a4 Untreated e-hGMSCs. b1-b4 e-hGMSCs treated with AA. c1-c4 e-hGMSCs treated with LPS-G. d1-d4 e-hGMSCs co-treated with LPS-G and AA. Scale bar $=10 \mu \mathrm{m}$
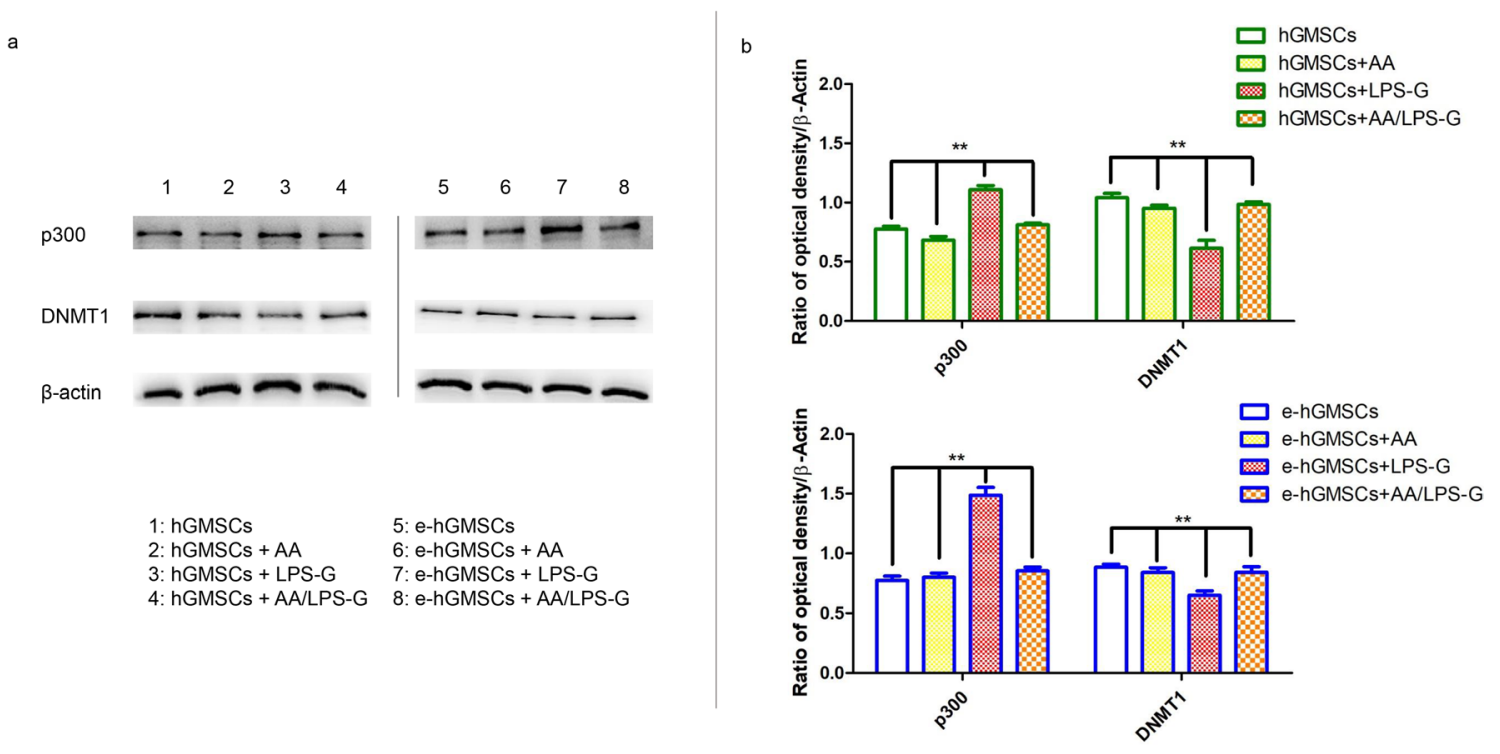

Fig. 7 Protein levels expression. a Western blotting-specific bands of P300 and DNMT1 expressions in hGMSCs and e-hGMSCs treated with LPS-G alone or in co-treatment of AA. b Bar graphs of den-

sitometric analysis normalized using $\beta$-actin. $(N=5)$. Values were expressed as mean $\pm \mathrm{SD}$ 
Fig. 8 ROS measurements. Images of live cells loaded with H2DCFDA and acquired by confocal microscopy: hGMSCs, hGMSCs + LPS-G, hGMSCs + AA, hGMSCs + AA/ LPS-G, e-hGMSCs, e-hGMSCs + LPS-G, e-hGMSCs + AA and e-hGMSCs + AA/LPS-G.

Scale bar $=20 \mu \mathrm{m} .(N=5)$.

Values were expressed as mean $\pm \mathrm{SD}$

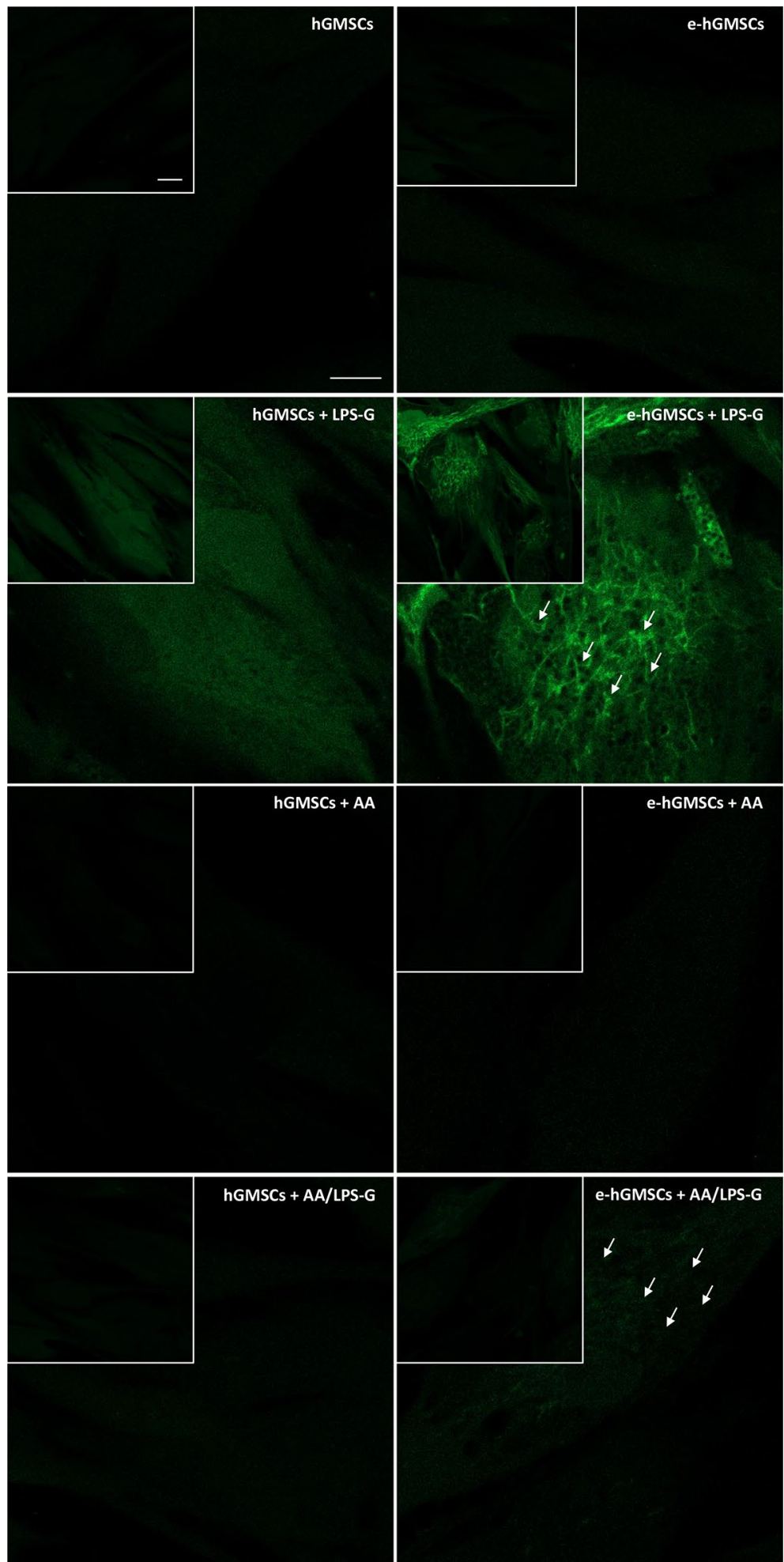

We then evaluated the changes in ROS levels upon LPS-G and AA treatment. Increased levels of ROS were observed in LPS-G-treated hGMSCs and e-hGMSCs, while AA reversed ROS toxicity. Our results are in line with an in vivo study where AA administration in a rat model of experimental periodontitis reduced oxidative stress (Aytekin et al. 2020), and staining revealed that AA markedly decreased the expression of p300 while increasing the expression of DNMT1, suggesting the anti-inflammatory role of AA. The results were further corroborated by western blot analysis. Further studies are required to better evaluate the molecular balance between DNMT1 activity and increased p300. 
also showed antioxidant effects in LPS-G-exposed hGMSCs and e-hGMSCs (Marconi et al. 2021).

Lastly, to support the idea that epigenetic modifications can regulate the expression of genes which influence the immune response, we analyzed the promoter methylation status of CCL5 and ICAMI genes in hGMSCs and e-hGMSCs after LPS-G and AA treatment. CCL5 is a member of the chemokine subfamily, and ICAMI is a member of the immunoglobulin supergene family. Both factors are known to be related to inflammatory response associated with oral pathological conditions (Sahingur and Yeudall 2015). Our data on promoter methylation analysis by pyrosequencing of $C C L 5$ and ICAMI genes did not reveal a significant modulation of CpG methylation after LPS-G and/or AA treatments in hGMSCs and e-hGMSCs. Schulz et al. studied the methylation profile of several inflammatory genes associated with periodontitis and reported that an altered methylation pattern was found only in CCL25 and IL17C genes (Schulz et al. 2016). In accord with this report, our results suggest that the protective role of AA against the inflammatory process triggered by LPS-G may not be connected to modulation in DNA methylation of CCL5 and ICAMI genes.

\section{Conclusion}

Our in vitro data demonstrated that LPS-G modulated the expression of epigenetic markers p300 and DNMT1, and enhanced ROS level in hGMSCs and e-hGMSCs. AA treatment ameliorated the epigenetic changes and reduced ROS. Further studies are warranted to evaluate the molecular mechanisms underlying AA which inhibit the inflammation-related epigenetic modifications caused by oral pathogens.

Supplementary Information The online version contains supplementary material available at https://doi.org/10.1007/s00418-021-02014-8.

Acknowledgements The present paper was supported by research funds of the University "G. d'Annunzio," Chieti-Pescara (OT/2019, FD/2020, GDM/2020). TSR would like to thank DST-FIST India for support to the Department of Biotechnology, Karpagam Academy of Higher Education (SR/FST/LS-1/2018/187; dated 26/12/2018).

Author contributions JP, FD, and OT initiated, designed, and supervised the project and wrote the article. GDM, SG, LF, YDR, FD, JP, FK, and VG performed the experiments and contributed to writing the article. GDM, SG, VG, and TSR analyzed and validated the obtained data. All authors reviewed the final version of the manuscript.

Funding Open access funding provided by Università degli Studi G. D’Annunzio Chieti Pescara within the CRUI-CARE Agreement.

Data availability Data are available from the corresponding author upon request.

\section{Declarations}

Conflict of interest The authors have no conflict of interest to declare.

Ethics approval The present study was approved by the ethical committee of the University "G. d'Annunzio" Chieti-Pescara.

Open Access This article is licensed under a Creative Commons Attribution 4.0 International License, which permits use, sharing, adaptation, distribution and reproduction in any medium or format, as long as you give appropriate credit to the original author(s) and the source, provide a link to the Creative Commons licence, and indicate if changes were made. The images or other third party material in this article are included in the article's Creative Commons licence, unless indicated otherwise in a credit line to the material. If material is not included in the article's Creative Commons licence and your intended use is not permitted by statutory regulation or exceeds the permitted use, you will need to obtain permission directly from the copyright holder. To view a copy of this licence, visit http://creativecommons.org/licenses/by/4.0/.

\section{References}

Aarabi G, Heydecke G, Seedorf U (2018) Roles of oral infections in the pathomechanism of atherosclerosis. Int J Mol Sci. https://doi. org/10.3390/ijms19071978

Aas JA, Paster BJ, Stokes LN, Olsen I, Dewhirst FE (2005) Defining the normal bacterial flora of the oral cavity. J Clin Microbiol 43(11):5721-5732. https://doi.org/10.1128/JCM.43.11.57215732.2005

Andrukhov O, Behm C, Blufstein A, Rausch-Fan X (2019) Immunomodulatory properties of dental tissue-derived mesenchymal stem cells: implication in disease and tissue regeneration. World J Stem Cells 11(9):604-617. https://doi.org/10.4252/wjsc.v11.i9. 604

Aytekin Z, Arabaci T, Toraman A, Bayir Y, Albayrak M, Ustun K (2020) Immune modulatory and antioxidant effects of locally administrated vitamin $\mathrm{C}$ in experimental periodontitis in rats. Acta Odontol Scand 78(6):425-432. https://doi.org/10.1080/00016357. 2020.1734656

Ballerini P, Diomede F, Petragnani N, Cicchitti S, Merciaro I, Cavalcanti M, Trubiani O (2017) Conditioned medium from relapsingremitting multiple sclerosis patients reduces the expression and release of inflammatory cytokines induced by LPS-gingivalis in THP-1 and MO3.13 cell lines. Cytokine 96:261-272. https://doi. org/10.1016/j.cyto.2017.04.022

Barachini S, Danti S, Pacini S, D’Alessandro D, Carnicelli V, Trombi L, Moscato S, Mannari C, Cei S, Petrini M (2014) Plasticity of human dental pulp stromal cells with bioengineering platforms: a versatile tool for regenerative medicine. Micron 67:155-168. https://doi.org/10.1016/j.micron.2014.07.003

Bianchi M, Pisciotta A, Bertoni L, Berni M, Gambardella A, Visani A, Russo A, de Pol A, Carnevale G (2017) Osteogenic differentiation of hDPSCs on biogenic bone apatite thin films. Stem Cells Int 2017:3579283. https://doi.org/10.1155/2017/3579283

Campos K, Gomes CC, Farias LC, Silva RM, Letra A, Gomez RS (2016) DNA methylation of MMP9 is associated with high levels of MMP-9 messenger RNA in periapical inflammatory lesions. J Endodontics 42(1):127-130. https://doi.org/10.1016/j.joen.2015. 10.002 
Chapple ILC, Matthews JB (2007) The role of reactive oxygen and antioxidant species in periodontal tissue destruction. Periodontol 2000 43:160-232. https://doi.org/10.1111/j.1600-0757.2006.00178.x

Diomede F, Zini N, Gatta V, Fulle S, Merciaro I, D’Aurora M, La Rovere RM, Traini T, Pizzicannella J, Ballerini P, Caputi S, Piattelli A, Trubiani O (2016) Human periodontal ligament stem cells cultured onto cortico-cancellous scaffold drive bone regenerative process. Eur Cell Mater 32:181-201. https://doi.org/10.22203/ ecm.v032a12

Diomede F, Thangavelu SR, Merciaro I, D’Orazio M, Bramanti P, Mazzon E, Trubiani O (2017) Porphyromonas gingivalis lipopolysaccharide stimulation in human periodontal ligament stem cells: role of epigenetic modifications to the inflammation. Eur J Histochem 61(3):2826. https://doi.org/10.4081/ejh.2017.2826

Diomede F, Marconi GD, Guarnieri S, D'Attilio M, Cavalcanti M, Mariggio MA, Pizzicannella J, Trubiani O (2019) A novel role of ascorbic acid in anti-inflammatory pathway and ROS generation in HEMA treated dental pulp stem cells. Materials (basel). https:// doi.org/10.3390/ma13010130

Diomede F, Marconi GD, Fonticoli L, Pizzicanella J, Merciaro I, Bramanti P, Mazzon E, Trubiani O (2020) Functional relationship between osteogenesis and angiogenesis in tissue regeneration. Int J Mol Sci. https://doi.org/10.3390/ijms21093242

Egger G, Liang G, Aparicio A, Jones PA (2004) Epigenetics in human disease and prospects for epigenetic therapy. Nature 429(6990):457-463. https://doi.org/10.1038/nature02625

Frei B, England L, Ames BN (1989) Ascorbate is an outstanding antioxidant in human-blood plasma. P Natl Acad Sci USA 86(16):6377-6381. https://doi.org/10.1073/pnas.86.16.6377

Fujisawa K, Hara K, Takami T, Okada S, Matsumoto T, Yamamoto N, Sakaida I (2018) Evaluation of the effects of ascorbic acid on metabolism of human mesenchymal stem cells. Stem Cell Res Ther 9(1):93. https://doi.org/10.1186/s13287-018-0825-1

Gugliandolo A, Diomede F, Cardelli P, Bramanti A, Scionti D, Bramanti P, Trubiani O, Mazzon E (2018) Transcriptomic analysis of gingival mesenchymal stem cells cultured on 3D bioprinted scaffold: a promising strategy for neuroregeneration. J Biomed Mater Res, Part A 106(1):126-137. https://doi.org/10.1002/jbm.a.36213

Hajishengallis G (2014) Immunomicrobial pathogenesis of periodontitis: keystones, pathobionts, and host response. Trends Immunol 35(1):3-11. https://doi.org/10.1016/j.it.2013.09.001

Hajishengallis G (2015) Periodontitis: from microbial immune subversion to systemic inflammation. Nat Rev Immunol 15(1):30-44. https://doi.org/10.1038/nri3785

Hajishengallis G, Darveau RP, Curtis MA (2012) The keystone-pathogen hypothesis. Nat Rev Microbiol 10(10):717-725. https://doi. org/10.1038/nrmicro2873

Kebschull M, Demmer RT, Papapanou PN (2010) “Gum bug, leave my heart alone!"-epidemiologic and mechanistic evidence linking periodontal infections and atherosclerosis. J Dent Res 89(9):879902. https://doi.org/10.1177/0022034510375281

Lee Chong T, Ahearn EL, Cimmino L (2019) Reprogramming the epigenome with vitamin C. Front Cell Dev Biol 7:128. https:// doi.org/10.3389/fcell.2019.00128

Li CH, Li B, Dong ZW, Gao L, He XN, Liao L, Hu CH, Wang QT, Jin Y (2014) Lipopolysaccharide differentially affects the osteogenic differentiation of periodontal ligament stem cells and bone marrow mesenchymal stem cells through Toll-like receptor 4 mediated nuclear factor kappa B pathway. Stem Cell Res Ther. https:// doi.org/10.1186/Scrt456

Libro R, Diomede F, Scionti D, Piattelli A, Grassi G, Pollastro F, Bramanti P, Mazzon E, Trubiani O (2016a) Cannabidiol modulates the expression of Alzheimer's disease-related genes in mesenchymal stem cells. Int J Mol Sci. https://doi.org/10.3390/ijms1 8010026
Libro R, Scionti D, Diomede F, Marchisio M, Grassi G, Pollastro F, Piattelli A, Bramanti P, Mazzon E, Trubiani O (2016b) Cannabidiol modulates the immunophenotype and inhibits the activation of the inflammasome in human gingival mesenchymal stem cells. Front Physiol 7:559. https://doi.org/10.3389/fphys.2016.00559

Lien E, Means TK, Heine H, Yoshimura A, Kusumoto S, Fukase K, Fenton MJ, Oikawa M, Qureshi N, Monks B, Finberg RW, Ingalls RR, Golenbock DT (2000) Toll-like receptor 4 imparts ligandspecific recognition of bacterial lipopolysaccharide. J Clin Invest 105(4):497-504. https://doi.org/10.1172/JCI8541

Liu R, Desta T, Raptis M, Darveau RP, Graves DT (2008) P. gingivalis and $E$. coli lipopolysaccharides exhibit different systemic but similar local induction of inflammatory markers. J Periodontol 79(7):1241-1247. https://doi.org/10.1902/jop.2008.070575

Marconi GD, Diomede F, Pizzicannella J, Fonticoli L, Merciaro I, Pierdomenico SD, Mazzon E, Piattelli A, Trubiani O (2020) Enhanced VEGF/VEGF-R and RUNX2 expression in human periodontal ligament stem cells cultured on sandblasted/etched titanium disk. Front Cell Dev Biol 8:315. https://doi.org/10.3389/ fcell.2020.00315

Marconi GD, Fonticoli L, Guarnieri S, Cavalcanti M, Franchi S, Gatta V, Trubiani O, Pizzicannella J, Diomede F (2021) Ascorbic acid: a new player of epigenetic regulation in LPS-gingivalis treated human periodontal ligament stem cells. Oxid Med Cell Longev 2021:6679708. https://doi.org/10.1155/2021/6679708

Martins MD, Jiao Y, Larsson L, Almeida LO, Garaicoa-Pazmino C, Le JM, Squarize CH, Inohara N, Giannobile WV, Castilho RM (2016) Epigenetic modifications of histones in periodontal disease. J Dent Res 95(2):215-222. https://doi.org/10.1177/0022034515611876

Mazzatenta A, Marconi GD, Zara S, Cataldi A, Porzionato A, Di Giulio C (2014) In the carotid body, galanin is a signal for neurogenesis in young, and for neurodegeneration in the old and in drugaddicted subjects. Front Physiol 5:427. https://doi.org/10.3389/ fphys.2014.00427

Mehrazarin S, Alshaikh A, Kang MK (2017) Molecular mechanisms of apical periodontitis: emerging role of epigenetic regulators. Dent Clin North Am 61(1):17-35. https://doi.org/10.1016/j.cden. 2016.08.003

Moore LD, Le T, Fan G (2013) DNA methylation and its basic function. Neuropsychopharmacol: off Publ Am Coll Neuropsychopharmacol 38(1):23-38. https://doi.org/10.1038/npp.2012.112

Nakano K, Wada K, Nomura R, Nemoto H, Inaba H, Kojima A, Naka S, Hokamura K, Mukai T, Nakajima A, Umemura K, Kamisaki Y, Yoshioka H, Taniguchi K, Amano A, Ooshima T (2011) Characterization of aortic aneurysms in cardiovascular disease patients harboring Porphyromonas gingivalis. Oral Dis 17(4):370-378. https://doi.org/10.1111/j.1601-0825.2010.01759.x

Papapanou PN, Susin C (2017) Periodontitis epidemiology: is periodontitis under-recognized, over-diagnosed, or both? Periodontol 2000 75(1):45-51. https://doi.org/10.1111/prd.12200

Pihlstrom BL, Michalowicz BS, Johnson NW (2005) Periodontal diseases. Lancet 366(9499):1809-1820. https://doi.org/10.1016/ S0140-6736(05)67728-8

Pisciotta A, Bertani G, Bertoni L, Di Tinco R, De Biasi S, Vallarola A, Pignatti E, Tupler R, Salvarani C, de Pol A, Carnevale G (2020) Modulation of cell death and promotion of chondrogenic differentiation by Fas/FasL in human dental pulp stem cells (hDPSCs). Frontiers in Cell and Developmental Biology 8:279. https://doi. org/10.3389/fcell.2020.00279

Pizzicannella J, Diomede F, Merciaro I, Caputi S, Tartaro A, Guarnieri S, Trubiani O (2018) Endothelial committed oral stem cells as modelling in the relationship between periodontal and cardiovascular disease. J Cell Physiol 233(10):6734-6747. https://doi.org/ $10.1002 / j \mathrm{cp} .26515$

Pizzicannella J, Diomede F, Gugliandolo A, Chiricosta L, Bramanti P, Merciaro I, Orsini T, Mazzon E, Trubiani O (2019) 3D printing 
PLA/Gingival stem cells/ EVs upregulate miR-2861 and -210 during osteoangiogenesis commitment. Int J Mol Sci. https://doi.org/ 10.3390/ijms20133256

Pizzicannella J, Fonticoli L, Guarnieri S, Marconi GD, Rajan TS, Trubiani O, Diomede F (2021) Antioxidant ascorbic acid modulates NLRP3 inflammasome in LPS-G treated oral stem cells through NFkappaB/Caspase-1/IL-1beta pathway. Antioxidants. https://doi. org/10.3390/antiox 10050797

Priyamvara A, Dey AK, Bandyopadhyay D, Katikineni V, Zaghlol R, Basyal B, Barssoum K, Amarin R, Bhatt DL, Lavie CJ (2020) Periodontal inflammation and the risk of cardiovascular disease. Curr Atheroscler Rep 22(7):28. https://doi.org/10.1007/ s11883-020-00848-6

Rajan TS, Scionti D, Diomede F, Grassi G, Pollastro F, Piattelli A, Cocco L, Bramanti P, Mazzon E, Trubiani O (2017) Gingival stromal cells as an in vitro model: cannabidiol modulates genes linked with amyotrophic lateral sclerosis. J Cell Biochem 118(4):819828. https://doi.org/10.1002/jcb. 25757

Sahingur SE, Yeudall WA (2015) Chemokine function in periodontal disease and oral cavity cancer. Front Immunol 6:214. https://doi. org/10.3389/fimmu.2015.00214

Schulz S, Immel UD, Just L, Schaller HG, Glaser C, Reichert S (2016) Epigenetic characteristics in inflammatory candidate genes in aggressive periodontitis. Hum Immunol 77(1):71-75. https://doi. org/10.1016/j.humimm.2015.10.007

Sinjari B, Pizzicannella J, D’Aurora M, Zappacosta R, Gatta V, Fontana A, Trubiani O, Diomede F (2019) Curcumin/liposome nanotechnology as delivery platform for anti-inflammatory activities via $\mathrm{NFkB/ERK/pERK} \mathrm{pathway} \mathrm{in} \mathrm{human} \mathrm{dental} \mathrm{pulp} \mathrm{treated} \mathrm{with}$ 2-HydroxyEthyl MethAcrylate (HEMA). Front Physiol 10:633. https://doi.org/10.3389/fphys.2019.00633

Socransky SS, Haffajee AD, Cugini MA, Smith C, Kent RL Jr (1998) Microbial complexes in subgingival plaque. J Clin Periodontol 25(2):134-144. https://doi.org/10.1111/j.1600-051x.1998.tb024 19. $\mathrm{x}$

Rajan TS, Giacoppo S, Diomede F, Bramanti P, Trubiani O, Mazzon E (2017) Human periodontal ligament stem cells secretome from multiple sclerosis patients suppresses NALP3 inflammasome activation in experimental autoimmune encephalomyelitis. Int $\mathbf{J}$ Immunopathol Pharmacol 30(3):238-252. https://doi.org/10.1177/ 0394632017722332

Trubiani O, Guarnieri S, Diomede F, Mariggio MA, Merciaro I, Morabito C, Cavalcanti MFXB, Cocco L, Ramazzotti G (2016) Nuclear translocation of $\mathrm{PKC}$ alpha isoenzyme is involved in neurogenic commitment of human neural crest-derived periodontal ligament stem cells. Cell Signal 28(11):1631-1641. https://doi.org/10. 1016/j.cellsig.2016.07.012

Vanden Berghe W, De Bosscher K, Boone E, Plaisance S, Haegeman $\mathrm{G}$ (1999) The nuclear factor-kappaB engages CBP/p300 and histone acetyltransferase activity for transcriptional activation of the interleukin-6 gene promoter. J Biol Chem 274(45):32091-32098. https://doi.org/10.1074/jbc.274.45.32091

Vapaatalo H, Mervaala E (2001) Clinically important factors influencing endothelial function. Med Sci Monitor Int Med J Exp Clin Res 7(5):1075-1085

Wei FL, Qu CY, Song TL, Ding G, Fan ZP, Liu DY, Liu Y, Zhang CM, Shi ST, Wang SL (2012) Vitamin C treatment promotes mesenchymal stem cell sheet formation and tissue regeneration by elevating telomerase activity. J Cell Physiol 227(9):3216-3224. https://doi.org/10.1002/jcp.24012

Wichnieski C, Maheshwari K, Souza LC, Nieves F, Tartari T, Garlet GP, Carneiro E, Letra A, Silva RM (2019) DNA methylation profiles of immune response-related genes in apical periodontitis. Int Endod J 52(1):5-12. https://doi.org/10.1111/iej.12966

Yussif NM, Aziz MAA, Rahman ARA (2016) Evaluation of the antiinflammatory effect of locally delivered vitamin $\mathrm{C}$ in the treatment of persistent gingival inflammation: clinical and histopathological study. J Nutr Metab. https://doi.org/10.1155/2016/2978741

Publisher's Note Springer Nature remains neutral with regard to jurisdictional claims in published maps and institutional affiliations. 